\title{
Rechtsprechung
}

\section{Kunstfreiheit eines Fotografen (Art. 5 Abs. 3 S. 1 GG) einerseits und all- gemeines Persönlichkeitsrecht der abgebildeten Person (Art. 2 Abs. 1 iVm Art. 1 Abs. 1 GG) andererseits bei Werken der Straßenfotografie}

\author{
BVerfG 1 BvR 2112/15 vom 8.2.2018
}

ECLI:DE:BVerfG:2018:rk20180208.1bvr211215

\section{Vorinstanz:}

KG Berlin, Beschluss vom 11.6.2015 - 10 U 119/14

\section{Tenor:}

Die Verfassungsbeschwerde wird nicht zur Entscheidung angenommen.

\section{Gründe}

I.

[1] Die Verfassungsbeschwerde betrifft die öffentliche Ausstellung einer künstlerischen Straßenfotografie ohne Einwilligung der abgebildeten Person.

[2] 1. Im Rahmen einer frei zugänglichen Ausstellung zum Thema "Ostkreuz: Westwärts. Neue Sicht auf Charlottenburg" wurden vor dem Gebäude des im Ausgangsverfahren neben dem Beschwerdeführer beklagten Ausstellungshauses auf 24 Ausstellungstafeln 146 Fotografien diversen Fotografen mit Bezug zum Stadtteil Berlin Charlottenburg an einer stark frequentierten Straße im gleichen Stadtteil ausgestellt. Eine von dem Beschwerdeführer stammende Fotografie zeigte eine Straßenszene, die unweit des Ausstellungsortes aufgenommen worden war. Im Mittelpunkt der Aufnahme steht die Klägerin des Ausgangsverfahrens (im Folgenden: Klägerin). Sie hält eine Handtasche in der einen sowie Plastiktüten in der anderen Hand und überquert an einer Ampel die Straße. Sie trägt ein Kleid mit Schlangenmuster, ihr Körper nimmt etwa ein Drittel des Bildes ein. Die Klägerin scheint in Richtung der Kamera zu blicken, ihr Gesicht ist gut erkennbar, obwohl die obere Kante des Fotos an ihrer Stirn endet. Im Hintergrund sind Gebäude, darunter ein Leihhaus, und einige andere Personen zu sehen, letztere jedoch wesentlich kleiner und unschärfer. Das Bild nahm die gesamte Fläche einer Ausstellungstafel $(120 \times 140 \mathrm{~cm})$ ein; in dieser Größe waren sieben Fotografien mit anderen Motiven ausgestellt.

[3] Der Beschwerdeführer hatte die Klägerin weder in Bezug auf die Aufnahme noch auf die Veröffentlichung der Fotografie um ihre Einwilligung gebeten. Auf ein anwaltliches Schreiben der Klägerin gab der Beschwerdeführer eine strafbewehrte Unterlassungserklärung ab.
[4] Auf die Klage der Klägerin verurteilte das Landgericht den Beschwerdeführer, ihr entstandene Anwaltskosten in Höhe von $795,46 € z u$ ersetzen. Soweit die Klägerin darüber hinaus eine Geldentschädigung und eine fiktive Lizenzgebühr verlangt hatte, wies es die Klage ab. Grundsätzlich sei die Einwilligung der fotografierten Person zur Veröffentlichung ihres Bildnisses gem. $\S 22$ S. 1 Kunsturhebergesetz (KUG) erforderlich. Zwar könne zugunsten des Beschwerdeführers davon ausgegangen werden, dass die Veröffentlichung dem höheren Interesse der Kunst iSd $\S 23$ Abs. 1 Nr. 4 KUG diene. Dem stehe jedoch ein berechtigtes Interesse der Klägerin nach § 23 Abs. 2 KUG entgegen. Denn es liege eine hinreichend schwerwiegende Beeinträchtigung ihres Persönlichkeitsrechts vor. Sie sei zwar in der Öffentlichkeit, aber bei einem rein privaten Vorgang fotografiert worden, der in ihre Privatsphäre falle. Ihr Bildnis sei über Wochen überlebensgroß an einer vielbefahrenen Straße der Öffentlichkeit präsentiert und sie so aus ihrer Anonymität gerissen worden. Es sei auch nicht auszuschließen, dass im Bekanntenkreis der Klägerin der Eindruck habe entstehen können, sie habe sich gegen Bezahlung fotografieren lassen. Ein Ersatz für immaterielle Schäden sei ihr dennoch nicht zuzuerkennen, da die Verletzung so schwer nicht wiege. Die Klägerin sei in einer gewöhnlichen Alltagssituation und (anders als von ihr selbst empfunden) auch nicht unvorteilhaft oder herabsetzend abgebildet. Die Abbildung entfalte keine "Prangerwirkung". Insbesondere entstehe nicht der Eindruck, sie komme aus dem Leihhaus.

[5] Mit angegriffenem Beschluss wies das Kammergericht die Berufung des Beschwerdeführers gem. § 522 Abs. 2 ZPO zurück. Selbst wenn davon auszugehen sei, dass das Aufstellen des Fotos unter $\S 23$ Abs. 1 Nr. 4 KUG falle, stünden der Zurschaustellung des Bildnisses jedenfalls berechtigte Interessen der Klägerin nach § 23 Abs. 2 KUG entgegen. Das Foto sei nicht im Rahmen einer Ausstellung als eines unter vielen, sondern großformatig, die gesamte Fläche einer Stelltafel einnehmend an einer der verkehrsreichsten Straßen der Stadt zur Straße gewandt präsentiert worden. Insofern sei die Klägerin einer breiten Masse als Blickfang ausgesetzt worden und nicht, wie in einer Kunstausstellung regelmäßig zu erwarten, lediglich der Betrachtung durch kunstinteressierte Besucher.

[6] Eine Anhörungsrüge des Beschwerdeführers blieb erfolglos.

[7] 2. Mit seiner Verfassungsbeschwerde rügt er eine Verletzung seiner Kunstfreiheit aus Art. 5 Abs. 3 S. 1 GG sowie seines Anspruchs auf rechtliches Gehör aus Art. 103 Abs. 1 GG. 
[8] 3. Die Verfassungsbeschwerde ist der Klägerin sowie der Senatsverwaltung für Justiz, Verbraucherschutz und Antidiskriminierung Berlin zugestellt worden. Die Klägerin hat von ihrem Äußerungsrecht Gebrauch gemacht. Die Akten des Ausgangsverfahrens wurden beigezogen.

\section{II.}

[9] Die Verfassungsbeschwerde wird nicht zur Entscheidung angenommen. Die Voraussetzungen des § 93a Abs. 2 BVerfGG für eine zwingende Annahme liegen nicht vor. Die Verfassungsbeschwerde hat keine grundsätzliche verfassungsrechtliche Bedeutung. Ihre Annahme ist auch nicht zur Durchsetzung der Rechte des Beschwerdeführers angezeigt.

[10] 1. Das Bundesverfassungsgericht hat die für die Beurteilung der Verfassungsbeschwerde maßgeblichen verfassungsrechtlichen Fragen bereits entschieden. Die verfassungsrechtlichen Anforderungen an das Verhältnis von Kunstfreiheit und allgemeinem Persönlichkeitsrecht sind in der Rechtsprechung des Bundesverfassungsgerichts geklärt (vgl. BVerfGE 67, 213 $<228>$; 75, $369<379$ f. >; 119, $1<26$ f. >; vgl. auch zur Pressefreiheit und der Veröffentlichung von Fotos aus dem Alltagsleben BVerfGE 101, $361<380$ ff. >; 120, $180<188$ f. >; BVerfG, Beschluss der 1. Kammer des Ersten Senats vom 21. August 2006 - 1 BvR 2606/04 u.a. -, NJW 2006, 3406; Beschluss der 1. Kammer des Ersten Senats vom 16. Juni 2008 - 1 BvR 17/08 -, juris, Rn. 17).

[11] 2. Die zulässige Verfassungsbeschwerde ist nicht begründet.

[12] Die Verurteilung des Beschwerdeführers zur Erstattung von Rechtsanwaltskosten, die wegen der Geltendmachung eines Anspruchs auf Unterlassung der Veröffentlichung des streitgegenständlichen Fotos entstanden sind, beeinträchtigt den Beschwerdeführer zwar in seiner Kunstfreiheit aus Art. 5 Abs. 3 S. 1 GG (a). Gegen deren Abwägung mit dem Recht am eigenen Bild ( $§ 12$ BGB) als Ausdruck des allgemeinen Persönlichkeitsrechts der Klägerin gem. Art. 2 Abs. 1 in Verbindung mit Art. 1 Abs. $1 \mathrm{GG}$ ist aber von Verfassungs wegen nichts zu erinnern (b).

[13] a) Unabhängig von der vom Bundesverfassungsgericht wiederholt hervorgehobenen Schwierigkeit, den Begriff der Kunst abschließend zu definieren (vgl. BVerfGE 30, $173<188$ f. $>$; $67,213<224$ ff. $>$ ), stellt das ausgestellte Foto ein Kunstwerk dar, nämlich eine freie schöpferische Gestaltung, in der Eindrücke, Erfahrungen und Erlebnisse des Künstlers durch das Medium einer bestimmten Formensprache, hier der Fotografie, zur Anschauung gebracht werden (vgl. BVerfGE 30, $173<188$ f. $>$; 67, 213 $<226>$; 75, $369<377>$; 119, $1<20$ f. >; 142, $74<103$ f. Rn. 89>). Dass das Foto ein unverfälschtes Abbild der Realität darstellt, steht dem nicht entgegen, da der Anspruch des Beschwerdeführers deutlich wird, diese Wirklichkeit künstlerisch zu gestalten. Es ist gerade Ziel der Straßenfotografie, die Realität unverfälscht abzubilden, wobei das spezifisch Künstlerische in der bewuss- ten Auswahl des Realitätsausschnitts und der Gestaltung mit fotografischen Mitteln zum Ausdruck kommt (vgl. Hildebrand, ZUM 2016, S. 305).

[14] Von der Kunstfreiheit ist nicht nur das Anfertigen der Fotografie, sondern auch deren Zurschaustellung im Rahmen einer öffentlich zugänglichen Ausstellung erfasst (vgl. BVerfGE 30, $173<189>$; 36, $321<331>$; 67, $213<224>$; 81, $278<292>$; 119, $1<21$ f. $>$; $142,74<96$ Rn. $68>$ ).

[15] b) Bei der Auslegung und Anwendung der gesetzlichen Vorschriften des § 1004 Abs. 1 S. 2 iVm § 823 Abs. 1 BGB, §§ 22 ff. KUG, Art. 2 Abs. 1 iVm Art. 1 Abs. 1 GG hat das Kammergericht Bedeutung und Tragweite der Kunstfreiheit des Beschwerdeführers aber hinreichend Rechnung getragen.

[16] aa) Auch wenn die Parteien in einem Zivilrechtsstreit, in dem es um den Konflikt von Kunstfreiheit und Persönlichkeitsrecht geht, um grundrechtlich geschützte Positionen streiten, handelt es sich um einen Rechtsstreit zwischen privaten Parteien, zu dessen Entscheidung in erster Linie die Zivilgerichte berufen sind (vgl. BVerfGE 119, $1<22>$ ). Sind bei der Auslegung und Anwendung einfachrechtlicher Normen mehrere Deutungen möglich, so verdient diejenige den Vorzug, die den Wertentscheidungen der Verfassung entspricht (vgl. BVerfGE 8, 210 $<221>$; 88, $145<166>$; 129, $78<102>$ ) und die die Grundrechte der Beteiligten möglichst weitgehend in praktischer Konkordanz zur Geltung bringt (vgl. BVerfGE 142, $74<101$ Rn. 82>). Der Einfluss der Grundrechte auf die Auslegung und Anwendung der zivilrechtlichen Normen ist nicht auf Generalklauseln beschränkt, sondern erstreckt sich auf alle auslegungsfähigen und -bedürftigen Tatbestandsmerkmale der zivilrechtlichen Vorschriften (vgl. BVerfGE 112, $332<358>$; 142, $74<101$ Rn. 82>)

[17] Dabei gibt das Grundgesetz den Zivilgerichten regelmäßig keine bestimmte Entscheidung vor. Die Schwelle eines Verstoßes gegen Verfassungsrecht, den das Bundesverfassungsgericht zu korrigieren hat, ist erst dann erreicht, wenn die Auslegung der Zivilgerichte Fehler erkennen lässt, die auf einer grundsätzlich unrichtigen Anschauung von der Bedeutung der Grundrechte beruhen, insbesondere vom Umfang ihres Schutzbereichs, und auch in ihrer materiellen Bedeutung für den konkreten Rechtsfall von einigem Gewicht sind, insbesondere weil darunter die Abwägung der beiderseitigen Rechtspositionen im Rahmen der privatrechtlichen Regelung leidet (vgl. BVerfGE 142, $74<101$ Rn. 83> mwN; stRspr).

[18] (1) Die Kunstfreiheit ist in Art. 5 Abs. 3 S. 1 GG zwar vorbehaltlos, aber nicht schrankenlos gewährleistet. Die Schranken ergeben sich insbesondere aus den Grundrechten anderer Rechtsträger, aber auch aus sonstigen Rechtsgütern mit Verfassungsrang (BVerfGE 142, 74 <101 f. Rn. 84> mwN; stRspr).

[19] Das durch Art. 2 Abs. 1 iVm Art. 1 Abs. 1 GG geschützte allgemeine Persönlichkeitsrecht ist ein Rechtsgut von Verfassungsrang, das der Kunstfreiheit Grenzen ziehen kann (vgl. 
BVerfGE 67, $213<228>$ ). Zu den anerkannten Inhalten des allgemeinen Persönlichkeitsrechts gehören das Verfügungsrecht über die Darstellung der eigenen Person, die soziale Anerkennung sowie die persönliche Ehre (vgl. BVerfGE 54, $148<153$ f.>; 99, $185<193>$; 114, $339<346>$; 119, $1<24>$ ). Ein allgemeines oder gar umfassendes Verfügungsrecht über die Darstellung der eigenen Person enthält Art. 2 Abs. 1 iVm Art. 1 Abs. 1 GG nicht (vgl. BVerfGE 101, $361<380>$ ).

[20] Für die Gewichtung der Belange des Persönlichkeitsschutzes wird neben den Umständen der Gewinnung der Abbildung, etwa durch Ausnutzung von Heimlichkeit oder beharrlicher Nachstellung, auch bedeutsam, in welcher Situation die betroffene Person erfasst und wie sie dargestellt wird (vgl. BVerfGE 120, $180<207>$ ). Das Gewicht der mit der Abbildung verbundenen Beeinträchtigungen des Persönlichkeitsrechts ist erhöht, wenn die visuelle Darstellung durch Ausbreitung von üblicherweise der öffentlichen Erörterung entzogenen Einzelheiten des privaten Lebens thematisch die Privatsphäre berührt. Gleiches gilt, wenn die betroffene Person nach den Umständen, unter denen die Aufnahme gefertigt wurde, typischerweise die berechtigte Erwartung haben durfte, nicht öffentlich abgebildet zu werden, etwa weil sie sich in einer durch räumliche Privatheit geprägten Situation, insbesondere einem besonders geschützten Raum, aufhält (vgl. BVerfGE 101, 361 $<384>$; BVerfG, Beschluss der 3. Kammer des Ersten Senats vom 9. Februar 2017 - 1 BvR 967/15 -, juris, Rn. 17). Dem Schutzanspruch des Persönlichkeitsrechts kann jedoch auch außerhalb der Voraussetzungen einer örtlichen Abgeschiedenheit ein erhöhtes Gewicht zukommen, so wenn die Abbildung den Betroffenen in Momenten der Entspannung oder des Sich-GehenLassens außerhalb der Einbindung in die Pflichten des Berufs und Alltags erfasst (vgl. BVerfGE 120, $180<207>$ ).

[21] (2) Allerdings zieht die Kunstfreiheit ihrerseits dem Persönlichkeitsrecht Grenzen. Um diese im konkreten Fall zu bestimmen, genügt es mithin im gerichtlichen Verfahren nicht, ohne Berücksichtigung der Kunstfreiheit eine Beeinträchtigung des Persönlichkeitsrechts festzustellen: Es bedarf der Klärung, ob diese Beeinträchtigung derart schwerwiegend ist, dass die Freiheit der Kunst zurückzutreten hat; eine geringfügige Beeinträchtigung oder die bloße Möglichkeit einer schwerwiegenden Beeinträchtigung reichen hierzu angesichts der hohen Bedeutung der Kunstfreiheit nicht aus. Lässt sich freilich eine schwerwiegende Beeinträchtigung des Persönlichkeitsrechts zweifelsfrei feststellen, so kann sie auch nicht durch die Kunstfreiheit gerechtfertigt werden (vgl. BVerfGE 67, $213<228>$; vgl. auch BVerfGE 30, $173<195>$; 75, $369<380>$; 119, $1<27>$ ).

[22] Die Lösung der Spannungslage zwischen Persönlichkeitsschutz und dem Recht auf Kunstfreiheit kann nicht allein auf die Wirkungen eines Kunstwerks im außerkünstlerischen Sozialbereich abheben, sondern muss auch kunstspezifischen Gesichtspunkten Rechnung tragen (vgl. BVerfGE 30, $173<195>$; 142, $74<102$ Rn. 85>). In der Interpretation eines Kunstwerks sind werkgerechte Maßstäbe anzulegen, dabei sind in der
Abwägung der Kunstfreiheit mit anderen Belangen strukturtypische Merkmale einer Kunstform zu berücksichtigen (vgl. BVerfGE 75, $369<378$ f.>).

[23] bb) Nach diesen Maßstäben ist das Kammergericht in der angegriffenen Entscheidung bei der Abwägung der Belange des Persönlichkeitsschutzes mit den Anforderungen dervom Landgericht in der vom Kammergericht implizit in Bezug genommenen erstinstanzlichen Entscheidung angesprochenen - Kunstfreiheit im Ergebnis den verfassungsrechtlichen Anforderungen gerecht geworden.

[24] Das Kammergericht hat die Bedeutung und Tragweite der Kunstfreiheit bei der Zuordnung des Bildnisses zum Anwendungsbereich des $\S 23$ Abs. 1 Nr. 4 KUG und in das Ergebnis seiner Abwägung im Rahmen von § 23 Abs. 2 KUG einbezogen (vgl. BVerfGE 120, $180<223>$ ) und ist dabei auch den Eigengesetzlichkeiten der Straßenfotografie gerecht geworden. Indem es die Schwere der Beeinträchtigung des Persönlichkeitsrechts der Klägerin aus der Art der Präsentation des Bildes als großformatigem Blickfang an einer öffentlichen Straße herleitet, hat das Kammergericht nicht verkannt, dass es mit der Kunstfreiheit nicht vereinbar wäre, ihren Wirkbereich von vornherein auf Galerien, Museen oder ähnliche räumlich begrenzte Ausstellungsorte zu begrenzen, sondern hat die besondere Persönlichkeitsverletzung der Klägerin durch die hervorgehobene Präsentation auf einer großformatigen Stelltafel an einer der verkehrsreichsten Straßen einer Millionenstadt zum zentralen Punkt seiner Abwägung gemacht. Damit hat das Kammergericht die ungestellte Abbildung von Personen ohne vorherige Einwilligung, welche strukturtypisch für die Straßenfotografie ist (vgl. Hildebrand, ZUM 2016, S. $305<309,311$ f.>), nicht generell unmöglich gemacht.

Diese Entscheidung ist unanfechtbar. 